\title{
Inhibition of Sestrin2 overexpression in diabetic cardiomyopathy ameliorates cardiac injury via restoration of mitochondrial function
}

\author{
XIAODAN ZHANG, XIAOYI DENG, HUIYU YE, ZHISHAN CHEN and WANGEN LI \\ Department of Endocrinology, The Second Affiliated Hospital of Guangzhou Medical University, \\ Guangzhou, Guangdong 510260, P.R. China
}

Received October 20, 2021; Accepted January 21, 2022

DOI: $10.3892 /$ etm.2022.11191

\begin{abstract}
Mitochondrial dysfunction-induced apoptosis plays a crucial role in the progression of diabetic cardiomyopathy (DCM). Sestrin2 is an important oxidative stress response protein and is involved in the maintenance of mitochondrial function, especially under stress. The aim of the present study was to investigate the role of Sestrin2 in DCM and to explore the underlying mechanisms. H9c2 cardiomyocytes were induced with high glucose (HG) medium (33 mmol/l glucose) for an in vitro DCM model. C57BL/6 mice were induced for the in vivo DCM model by intraperitoneal streptozotocin injection. $\mathrm{H} 9 \mathrm{c} 2$ cardiomyocytes were exposed to $\mathrm{HG}$ and infected with lentiviruses to express Sestrin2 short hairpin RNA (shRNA). The study found that cell viability and mitochondrial function were impaired while cell apoptosis and oxidative stress were increased in DCM. Sestrin2 was significantly upregulated in myocardial tissues of DCM mice and $\mathrm{H} 9 \mathrm{c} 2$ cardiomyocytes in HG conditions. Downregulation of Sestrin2 increased cell viability, decreased cell apoptosis, and attenuated oxidative stress in $\mathrm{H} 9 \mathrm{c} 2$ cells exposed to HG. Moreover, HG-induced mitochondrial injury was alleviated by Sestrin 2 silencing. In conclusion, our finding indicated that the inhibition of enhanced Sestrin2 expression ameliorates cardiac injury in DCM, which might be largely attributed to the restoration of mitochondrial function.
\end{abstract}

\section{Introduction}

Diabetes mellitus (DM) is frequently complicated by cardiac dysfunction. Diabetes can affect cardiac structure and

Correspondence to: Dr Wangen Li, Department of Endocrinology, The Second Affiliated Hospital of Guangzhou Medical University, 250 East Changgang Road, Haizhu, Guangzhou, Guangdong 510260, P.R. China

E-mail: liwg660@126.com

Key words: diabetes mellitus, diabetic cardiomyopathy, mitochondrial function, oxidative stress, apoptosis function in the absence of high blood pressure and coronary artery disease, a condition known as diabetic cardiomyopathy (DCM). DCM increases the risk of heart failure and is one of the major causes of death in both type 1 DM (T1DM) and type 2 DM (T2DM) (1). The pathogenesis of DCM is closely related to hypoxia. Hyperglycemia-induced overproduction of superoxides by the mitochondrial election-transport chain is considered to be the common pathway. Mitochondrial dysfunction, oxidative stress and the resulting increased ROS generation appear to be key players in the development of $\operatorname{DCM}(2)$.

Sestrins (Sesns) family proteins are highly conserved stress-induced proteins, which are widely expressed in mammals and respond to a variety of environmental stresses (3). Sestrin2 (Sesn2, also termed Hi95) is an important member of the Sesn proteins, whose expression is relatively low in resting cells. High abundance of Sestrin 2 is mainly induced by metabolic stresses such as hypoxia, DNA lesions, oxidative stress and endoplasmic reticulum stress (4). Previously, research on Sestrin 2 mainly focused on obesity-associated metabolic diseases and age-related diseases. In addition, the role of Sestrin2 in atherosclerotic and cardiac diseases is becoming a concern (4). Nevertheless, research in DCM is rarely seen. It was previously reported that Sestrin2 helped to protect whole cellular energy metabolism and maintain mitochondrial function (5). Overexpression of Sestrin 2 could reduce ROS accumulation, maintain mitochondrial membrane potential, reduce ATP depletion, restore mitochondrial DNA, and ultimately, reduce cellular apoptosis (5).

Considering the protective role of Sestrin 2 in maintaining mitochondrial function, especially under stress and the pathogenesis mechanism including hypoxia, enhanced oxidative stress and mitochondrial dysfunction in DCM, it has been speculated that Sestrin 2 and the related pathways may play important roles in the development of DCM. The aim of this study was to investigate the effects of Sestrin2 in DCM and to explore the underlying mechanisms.

\section{Materials and methods}

Cell culture. The rat cardiomyoblast cell line, H9c2 cells (ATCC: CRL-1446), and 293T cells (ATCC: CRL-3216) were 
purchased from The Cell Bank of Type Collection of Chinese Academy of Sciences. Cells were cultured with low-glucose Dulbecco's modified essential medium (DMEM; Gibco; Thermo Fisher Scientific, Inc.) supplemented with $10 \%$ fetal bovine serum (FBS; Gibco; Thermo Fisher Scientific, Inc.), $1 \%$ penicillin-streptomycin solution (Gibco; Thermo Fisher Scientific, Inc.) and incubated with a humidified atmosphere of $5 \% \mathrm{CO}_{2}$ at $37^{\circ} \mathrm{C}$. The cells were sub-cultured every 2-3 days at a ratio of $1: 3$ or subjected in subsequent experiments at a $70-80 \%$ confluence.

Construction of Sestrin2 shRNA lentivirus. To construct a Sestrin 2 silencing lentiviral vector, three candidate sequences of Sestrin2 small hairpin RNA (shRNA) lentiviral vectors targeting rat Sestrin 2 mRNA were designed and synthesized by Invitrogen; Thermo Fisher Scientific, Inc. The shRNAs were constructed into the lentivirus expression vector using a lentivirus expressing system (HanBio Biotechnology Co., Ltd.) according to the manufacturer's instructions. Recombinant lentiviruses were produced by transfecting $293 \mathrm{~T}$ cells with the lentiviral expression plasmid. At $24 \mathrm{~h}$ prior to transfection, 293T cells were cotransfected with plasmid containing Sestrin 2 shRNA $(10 \mu \mathrm{g})$ and lentiviral packaging vector $(15 \mu \mathrm{g})$. Cells were then incubated in DMEM with 10\% FBS for $48 \mathrm{~h}$ at $37^{\circ} \mathrm{C}$, followed by harvesting, centrifugation $\left(50,000 \mathrm{x}\right.$ g at $4^{\circ} \mathrm{C}$ for $\left.2 \mathrm{~h}\right)$ and filtration. The Sestrin 2 shRNA targeting sequence (5'-GCAGAGACCCATTGAACAACT-3') was the most effective one and was used in subsequent experiments. The amplified virus with a multiplicity of infection of 50 was transfected to $\mathrm{H} 9 \mathrm{c} 2$ cells. The cells were treated with a reagent as indicated for further experiments after $48 \mathrm{~h}$. The empty vector was used as a negative control. Puromycin at a final concentration of $5 \mu \mathrm{g} / \mathrm{ml}$ was added to the medium $48 \mathrm{~h}$ after transfection to select for purely transfected cells. Cells were subsequently cultured every $2-3$ days at a ratio of 1:3 for 2-3 generations for stable constructions and the infection efficiency was assessed using western blot analysis.

Cell treatments. H9c2 cells were cultured in 6-well plates and were randomly divided into four groups: normal glucose control group (C; $5.5 \mathrm{mM}$ glucose), high glucose (HG) group (G; 33 mM glucose), Sestrin2 small hairpin RNA (shRNA) group (Sesn2 shRNA; $33 \mathrm{mM}$ glucose), and negative control group (NC; $33 \mathrm{mM}$ glucose). The cells were treated with the corresponding medium for $48 \mathrm{~h}$.

Animals and treatments. Male mice (C57BL/6; 8 weeks old) were purchased from Beijing Weitong Lihua Experimental Animal Technology Co., Ltd. Mice were housed in a temperature- $\left(22 \pm 1^{\circ} \mathrm{C}\right)$ and humidity-controlled $(50 \pm 10 \%)$ environment and maintained in a 12-h light/dark cycle. Free access to food and water was provided. After acclimatization for 1 week, mice were randomly divided into two groups: Control $(n=5)$ and DM $(n=8)$ groups. Diabetes was induced in mice in the DM group by intraperitoneal injection of streptozotocin (STZ; MilliporeSigma) dissolved in $0.1 \mathrm{ml}$ citrate buffer ( $\mathrm{pH} 4.5$ ) at a dose of $50 \mathrm{mg} / \mathrm{kg}$ for five consecutive days. The equivalent volume of citrate buffer was administered to mice in the control group. The blood sample, 0.1-0.2 ml each time, was obtained through the tail vein. At one week after the course of
STZ administration, mice with random blood glucose levels $>16.7 \mathrm{mmol} / \mathrm{l}$ were considered to be diabetic. After 8 weeks, the mice were euthanized with $4 \%$ isoflurane, and then sacrificed by cervical dislocation. The hearts were immediately harvested for subsequent experiments. Blood samples and myocardial tissues were collected.

All animal procedures were approved by the Ethics Committee of the Second Affiliated Hospital of Guangzhou Medical University (approval no.: 20200407). All animal studies were performed in accordance with the National Institutes of Health Guide for the Care and Use of Laboratory animals.

Cell viability. H9c2 cells were seeded in 96-well plates at a density of 5,000 cells/well. Viability was measured using the CellTiter $96^{\circledR}$ AQueous One Solution Assay kit (MTS) (Promega Corporation). Culture medium was replaced with complete medium (100 $\mu \mathrm{l} /$ well) following treatment. Then the medium was replaced with $10 \mu \mathrm{l}$ MTS assay reagent per well and incubated for $4 \mathrm{~h}$ at $37^{\circ} \mathrm{C}$. A microplate reader was used to detect the optical density (OD) at $490 \mathrm{~nm}$, according to the manufacturer's instructions.

Enzyme-linked immunosorbent assay (ELISA). H9c2 cell samples were collected at $1,000 \mathrm{~g}$ for $10 \mathrm{~min}$ and used for measurement of caspase-3 and cytochrome $c$ by ELISA using commercial kits (Nanjing Jiancheng Biological Engineering Research Institute), following the manufacturer's protocols.

Western blot analysis. Briefly, total protein of the cell and heart tissue were isolated and prepared, using the RIPA-PMSF lysis buffer (Beijing Solarbio Science \& Technology) and quantified with a BCA kit (Servicebio), followed by loading $\sim 40 \mu \mathrm{g}$ protein per lane. The samples were separated on $10 \%$ SDS-PAGE and then transblotted onto polyvinylidene fluoride (PVDF) membranes. PVDF membranes were blocked with $5 \%$ non-fat milk at room temperature for $2 \mathrm{~h}$. Samples were incubated with primary antibodies against Sestrin2 (catalog no. DF12003; 1:800; Affinity Bioscience) and GAPDH (catalog no. AF7021; 1:800; Affinity Bioscience) overnight at $4^{\circ} \mathrm{C}$. Following washes, the membranes were incubated with the goat anti-rabbit HRP-linked secondary antibody (catalog no. GB23303; 1:3,000; Wuhan Servicebio Technology Co., Ltd.) for $1 \mathrm{~h}$ at room temperature. The blots were visualized with an enhanced chemiluminescence detection system (Hangzhou Fude Biological Technology). ImageJ software (ImageJ 64-bit Java 1.8.0_112) was used to measure the gray-scale value of each band.

Reverse transcription-quantitative PCR (RT-qPCR). Total RNA was extracted from the cultured cells with TRIzol ${ }^{\circledR}$ reagent (Invitrogen; Thermo Fisher Scientific, Inc.), according to the manufacturer's instructions. The purity and quantity of RNA samples were determined using Epoch $^{\text {TM }}$ microplate spectrophotometer (BioTek Instruments, Inc.). Total RNA was reverse transcribed into cDNA according to the instructions of PrimeScript $^{\text {TM }}$ RT master mix (Takara Bio, Inc.). qPCR was performed using Applied Biosystems ${ }^{\mathrm{TM}}$ PowerUp $^{\mathrm{TM}} \mathrm{SYBR}^{\mathrm{TM}}$ Green mix (invitrogen). The reaction volume total was $30 \mu \mathrm{l}$ and PCR was performed with LightCycler ${ }^{\circledR} 480$. The results were analyzed by the $2^{-\Delta \Delta \mathrm{Cq}}$ method (6). Primer sequences used 
are shown as follows: Sestrin2 forward, 5'-GACCATGGCTA CTCGCTGAT-3' and reverse, 5'-CCAAAGACGCAGTGG ATGTA-3'; $\beta$-actin forward, 5'-AGGGAAATCGTGCGTGA CAT-3' and reverse, 5'-GAACCGCTCATTGCCGATAG-3'.

Histological and immunofluorescence analysis. Myocardial tissues were gathered and fixed in $4 \%$ phosphate-buffered paraformaldehyde overnight at room temperature. Paraffin-embedded tissues were sectioned at a thickness of $5 \mu \mathrm{m}$ for staining with hematoxylin and eosin (H\&E) for $3 \mathrm{~min}$ or Masson's trichrome for $5 \mathrm{~min}$ at room temperature. The histological changes of the myocardium and collagen deposition were imaged using a light microscope (Olympus; magnification, $\mathrm{x} 400$ ).

TUNEL assay. Myocardial apoptosis was detected using TUNEL assay. The heart tissue samples were fixed in $4 \%$ paraformaldehyde at room temperature overnight, embedded in paraffin and sectioned into 5- $\mu \mathrm{m}$ slices. Paraffin sections were dehydrated, dewaxed, and digested at room temperature for 8 min. An in situ cell detection kit (Roche Applied Science) was used to identify the apoptotic cells. The TUNEL-positive cells were observed and counted under a fluorescence microscope (Olympus). Left ventricle samples were also stained with primary antibodies against Sestrin2 (catalog no. 8487S; 1:200; Cell Signaling Technology, Inc.) at $4^{\circ} \mathrm{C}$ overnight, followed by the secondary antibody (catalog no. GB23303; 1:3,000; Wuhan Servicebio Technology Co.,Ltd.). The sections were then stained with 3,3'-diaminobenzidine and a fluorescence microscope (Olympus) was used to obtain images of the tissues.

Cell apoptosis assay. H9c2 cells were inoculated into a 6-well plate at a concentration of $1 \times 10^{6}$ cells/well, and cultured in a humidified environment containing $5 \% \mathrm{CO}_{2}$ at $37^{\circ} \mathrm{C}$ for $24 \mathrm{~h}$. Next, a ReadiDrop ${ }^{\mathrm{TM}}$ dual propidium iodide (PI)/fluorescein isothiocynate-conjugated (FITC) Annexin V commercial staining kit (Bio-Rad) was used to measure the cell apoptosis rate, according to the manufacturer's instructions. The change in apoptosis was analyzed with a flow cytometer (FACSCanto; BD Biosciences) and FLOWJO (version 10; BD Biosciences) after staining.

Measurement of intracellular ROS generation. For the detection of intracellular ROS generation, the indicated cells were incubated with 2',7'-dichlorodihydrofluorescein diacetate (DCFH-DA; Sigma-Aldrich) for $15 \mathrm{~min}$ at $37^{\circ} \mathrm{C}$. To determine the ROS production, the fluorescence intensity was measured by flow cytometry (FACSCanto) and FLOWJO (version 10).

Measurement of antioxidant and oxidant levels. The Cell Mitochondria Isolation Kit (Beijing Solarbio Science \& Technology) was used to isolate the mitochondria of $\mathrm{H} 9 \mathrm{c} 2$ cells. The activities of superoxide dismutase (SOD), mitochondrial SOD, and malondialdehyde (MDA) were determined with the corresponding detection kit according to the manufacturer's instructions (Nanjing Jiancheng Biotechnology Institute).

Measurement of mitochondrial membrane potential. JC-1 was broadly used for observing mitochondrial membrane potential and identifying fluorescence characteristic change from green
$(530 \mathrm{~nm})$ to red $(590 \mathrm{~nm})$ depending on the mitochondrial membrane potential. A red/green fluorescence intensity ratio of JC-1 indicated a decrease in depolarized mitochondria due to disruption of red fluorescent J-aggregates (Beyotime Biotechnology). H9c2 cells were collected, JC-1 working solution was added, and the cells were cultured at $37^{\circ} \mathrm{C}$ for $30 \mathrm{~min}$. Imaging Buffer solution was added, and the cells were analyzed by flow cytometry.

Intracellular $\mathrm{Ca}^{2+}$ measurement. The cells were collected and loaded with Fluo 3-AM (2 M) in the HEPES-buffered salt solution (HBSS) $\left(\mathrm{NaCl} 137 \mathrm{mM}, \mathrm{KCl} 2.7 \mathrm{mM}, \mathrm{NaH}_{2} \mathrm{PO}_{4} 0.4 \mathrm{mM}\right.$, $\mathrm{CaCl}_{2} 0.9 \mathrm{mM}, 0.5 \mathrm{mM} \mathrm{MgCl}, 10 \mathrm{mM}$ HEPES, and $5.5 \mathrm{mM}$ glucose, $\mathrm{pH}$ 7.4) for $30 \mathrm{~min}$ at $37^{\circ} \mathrm{C}$. The cells were resuspended, and then incubated with the Fluo-3/AM ( $3 \mathrm{mM})$ for $30 \mathrm{~min}$ at $37^{\circ} \mathrm{C}$. Subsequently, protein blocking was completed with $0.2 \% \mathrm{BSA}$ which was flushed with $\mathrm{Ca}^{2+}$-free HBSS three times. Finally, flow cytometry was used to detect intracellular calcium levels at an excitation wavelength of $488 \mathrm{~nm}$ and an emission wavelength of $525 \mathrm{~nm}$.

Measurement of MPTP opening. The mitochondrial permeability transition pore (MPTP) opening of H9c2 cells was measured by the MPTP Assay Kit (Beyotime Biotechnology). After 0, 24, and $36 \mathrm{~h}$ compression, the cells were collected. Subsequently, $500 \mu 1$ preheated cleaning solution (Reagent A) and isopyknic working solution containing neutralization and staining solution (Reagent B) were added into the cell suspension. The cell suspension was then mixed gently and fully and incubated for $20 \mathrm{~min}$ at $37^{\circ} \mathrm{C}$ in the dark. Subsequently, the samples were resuspended in Reagent A and analyzed by flow cytometry.

Measurement of ATP levels. The level of ATP in H9c2 cells was measured by an ATP assay kit (Beyotime Biotechnology). $\mathrm{H} 9 \mathrm{c} 2$ cells were rinsed with PBS and lysed with lysis buffer on ice to collect the lysate and centrifuged for $4 \mathrm{~min}$ at $12,000 \mathrm{x} \mathrm{g}$ at $4^{\circ} \mathrm{C}$. Sample supernatant was then added containing $100 \mu \mathrm{l}$ ATP detection solution and mixed vibration. A multifunctional microplate reader (VICTOR X5; PerkinElmer, Inc.) was used to determine the ATP content of the sample.

Statistical analysis. Data were analyzed using SPSS 16.0 (SPSS, Inc.) statistical software and presented as the mean \pm standard deviation (SD). One-way analysis of variance (ANOVA) test and post hoc analysis with a Bonferroni test were used for multi-group comparisons. Unpaired Student's t-test was used to compare two groups. Two-sided $\mathrm{P}<0.05$ was considered to indicate a statistically significant difference.

\section{Results}

Sestrin 2 is significantly upregulated in DCM conditions. The characteristics of DCM were found in the left ventricular sections of DM mice by H\&E and Masson staining. The results showed that the myocardial fibers were arranged disorderly, the nucleus sizes became different and more vacuoles and collapse of myofibers were identified in mice in the DM group. Apoptotic cells were identified by the TUNEL assay. Brown-stained nuclei indicated apoptotic 
A

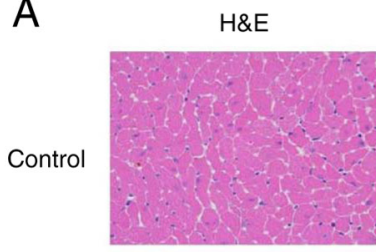

DM

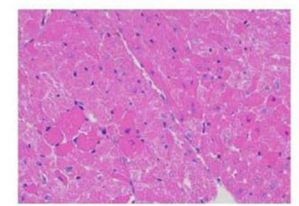

Masson

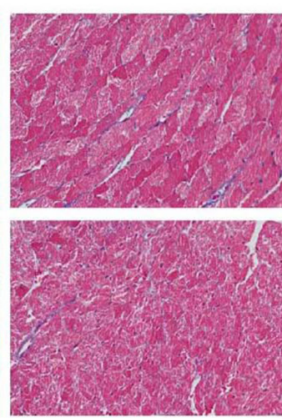

TUNEL

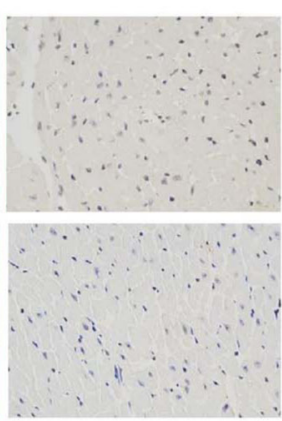

B
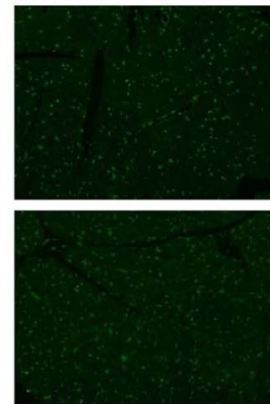

Control
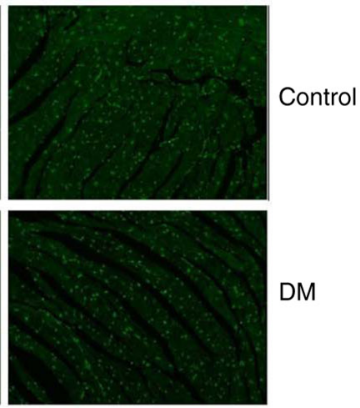

C
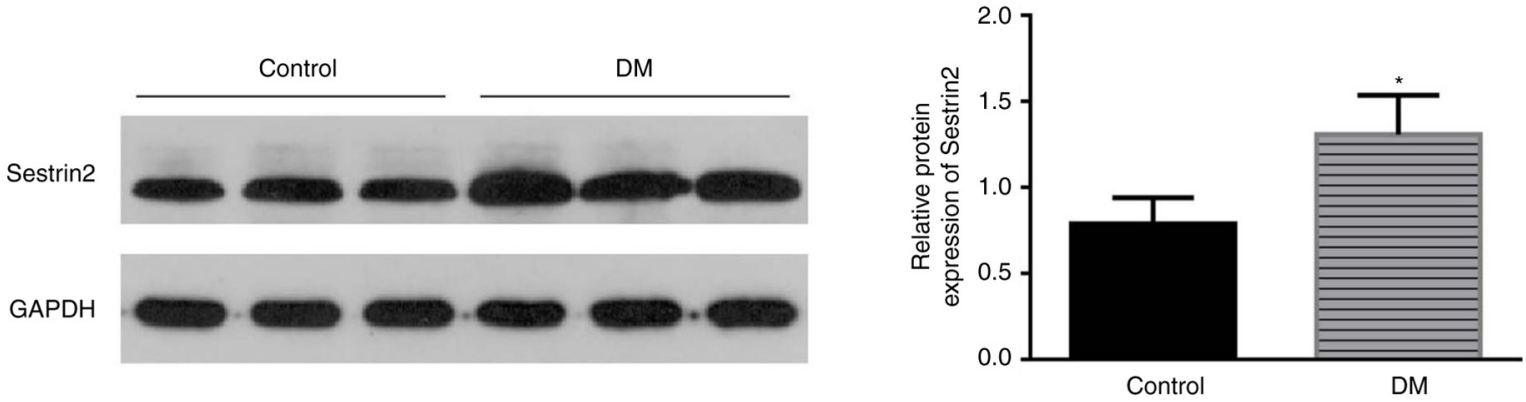

Figure 1. The expression levels of Sestrin2 were increased in myocardial tissues in DM mice. (A) Typical images of myocardial tissue sections stained with H\&E, Masson's trichrome, and TUNEL (magnification, $\mathrm{x} 400$ ). The expression levels of Sestrin2 in myocardial tissues in DM mice and controls were detected by an (B) immunofluorescence assay and $(C)$ western blotting. Data are presented as mean $\pm \mathrm{SD}(\mathrm{n}=3)$. " $\mathrm{P}<0.001$ vs. Control group.

A

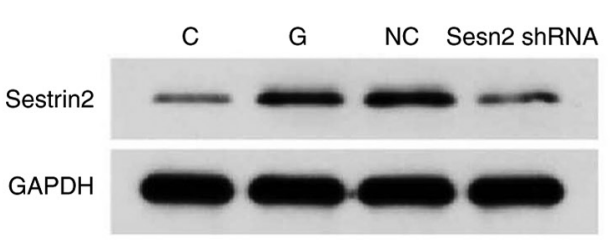

B

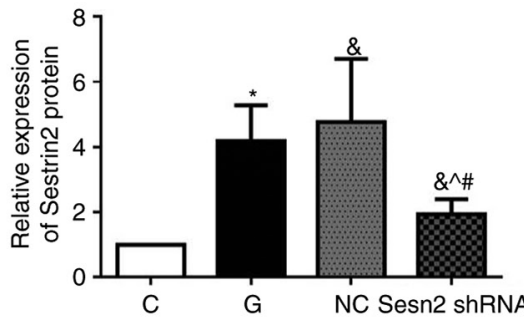

C

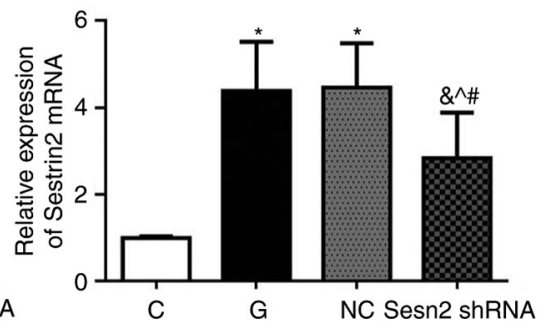

Figure 2. The expression levels of Sestrin2 were increased in H9c2 cardiomyocytes under hyperglycemic conditions compared with controls. (A) Relative protein expression levels of Sestrin2 were measured via western blotting. (B) Results of gray-scale analysis of protein expression. Data are presented as mean $\pm \mathrm{SD}(\mathrm{n}=3)$. (C) Sestrin 2 mRNA expression was detected by RT-qPCR analysis. Data are presented as mean $\pm \mathrm{SD}(\mathrm{n}=3)$. ${ }^{*} \mathrm{P}<0.01$ vs. Control group. ${ }^{\&} \mathrm{P}<0.05$ vs. Control group. ${ }^{\wedge} \mathrm{P}<0.05$ vs. HG group. ${ }^{\#} \mathrm{P}<0.05$ vs. Negative control group. Sesn2, Sestrin2; C, control group; G, HG group; NC, negative control; shRNA, small hairpin RNA; HG, high glucose.

cells, and blue-green or tan shades indicated non-apoptotic cells in diabetic mouse hearts (Fig. 1A). Few apoptotic cells were observed in the control group, while increased apoptotic cardiomyoblasts were observed in DM mice (Fig. 1C). To assess the potential effect of Sestrin2 in DCM, Sestrin2 expressions in DCM models were measured by immunofluorescence assay (Fig. 1B), western blotting and RT-qPCR (Fig. 1C). The results revealed that the expression of Sestrin2 was significantly increased in myocardial tissues of DM mice $(\mathrm{P}<0.001$, Fig. 1$)$ and $\mathrm{H} 9 \mathrm{c} 2$ cardiomyocytes in $\mathrm{HG}$ conditions $(\mathrm{P}<0.01$, Fig. 2).

Downregulation of Sestrin2 increases cell viability and decreases cell apoptosis induced by HG. Sestrin2 shRNA lentivirus was constructed for Sestrin 2 silencing. The efficiency of siRNA-mediated inhibition of Sestrin 2 was evaluated by detecting Sestrin 2 protein by western blot analysis and mRNA levels by RT-qPCR. Compared with the NC group, levels of protein expression and mRNA of Sestrin2 were significantly decreased in the Sesn 2 shRNA group $(\mathrm{P}<0.05)$ (Fig. 2).

The cell viabilities were measured by MTS analysis. As shown in Fig. 3A, cell viabilities were decreased greatly in $\mathrm{G}$ group compared to $\mathrm{C}$ group $(\mathrm{P}<0.001)$. The downregulation of Sestrin2 significantly increased cell viability of H9c2 cells, compared with the $\mathrm{G}$ and $\mathrm{NC}$ groups $(\mathrm{P}<0.001)$. To evaluate the possible effects of downregulation of Sestrin2 on HG-treated H9c2 cell apoptosis, the apoptotic rates in the four groups were determined by flow cytometry using V-FITC/PI staining. As shown in Fig. 3B and C, despite the high PI cells (about 10\% of the total) in Q1 in flow cytometry, the apoptotic rate was very low in $\mathrm{C}$ group, while it increased significantly in $\mathrm{G}$ group $(\mathrm{P}<0.001)$. The apoptotic rate was significantly decreased after Sestrin2 shRNA transfection, compared with the $\mathrm{G}$ and $\mathrm{NC}$ groups $(\mathrm{P}<0.001)$. These results indicated that the downregulation of Sestrin2 could provide cardioprotective 
A

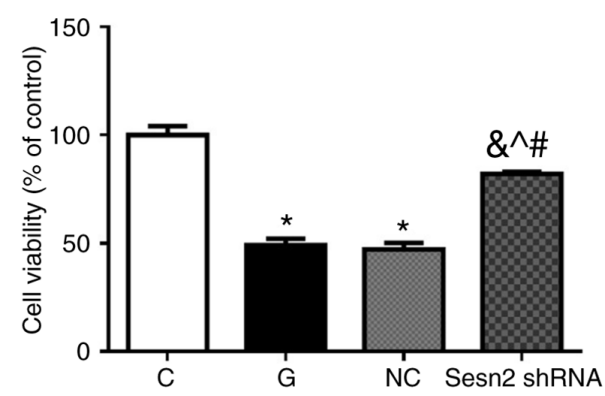

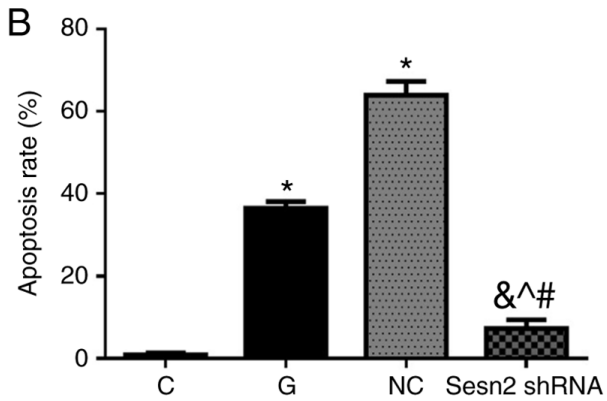

C
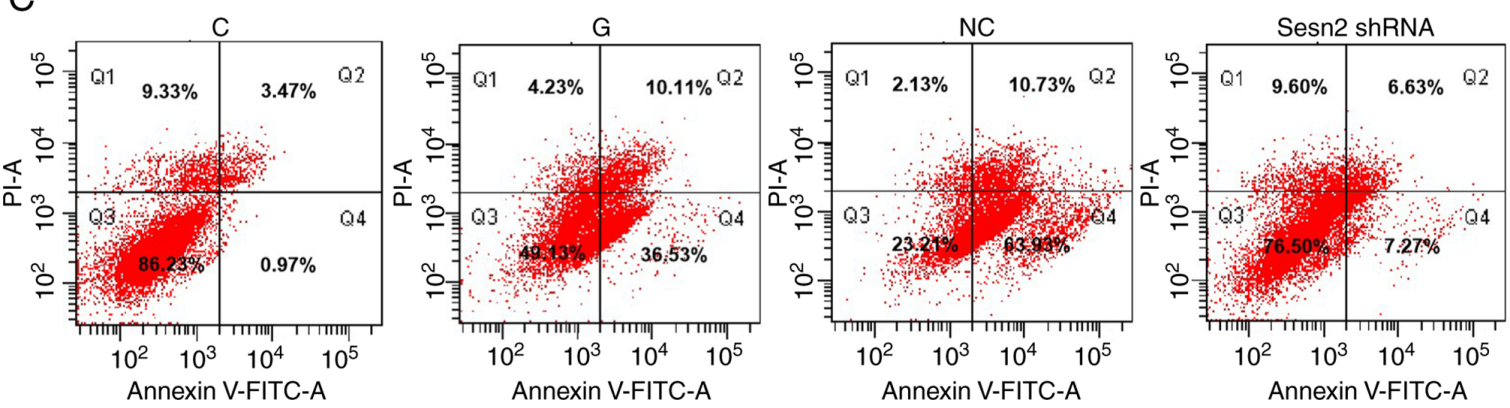

Figure 3. Sestrin2 inhibition significantly increases cell viability and decreases cell apoptosis in H9c2 cells under hyperglycemic conditions. (A) Cell viability was determined by MTS assay after cell treatments. (B) The apoptotic rate of H9c2 cells was detected via flow cytometry. (C) Quantitative analysis of the apoptotic rate. Data are presented as mean $\pm \mathrm{SD}(\mathrm{n}=3)$. ${ }^{*} \mathrm{P}<0.001$ vs. Control group. ${ }^{\circledR} \mathrm{P}<0.01$ vs. Control group. ${ }^{\wedge} \mathrm{P}<0.001$ vs. HG group. ${ }^{\sharp} \mathrm{P}<0.001$ vs. Negative control group. Sesn2, Sestrin2; C, control group; G, HG group; NC, negative control; shRNA, small hairpin RNA, HG, high glucose.

effects in hyperglycemic conditions by attenuating cardiomyocyte injury and apoptosis. Specifically, significant populations of high PI cells in Q1 in flow cytometry were identified in repetitive experiments performed by different members of the research group, without observing obvious cell necrosis during cell culture. Since a common cause for this situation is a mechanical lesion, the HG concentration in the culture medium may lead to a higher permeability of PI through the cell membrane.

Downregulation of Sestrin2 attenuates oxidative stress in $H 9 c 2$ cells exposed to $H G$. Overwhelming oxidative stress is the key procedure of DCM. Excessive ROS production can induce mitochondrial dysfunction of cardiomyocytes, thereby causing apoptosis (7). DCFH-DA was used to detect cellular oxidative stress. As shown in Fig. 4A, intracellular ROS production was markedly increased in $\mathrm{H} 9 \mathrm{c} 2$ cells under HG conditions. However, the downregulation of Sestrin2 significantly reduced ROS level in H9c2 cells, compared with $\mathrm{G}$ and $\mathrm{NC}$ groups $(\mathrm{P}<0.001)$. In addition, oxidant (MDA) and antioxidant levels (SOD and mitochondrial SOD) were determined. As shown in Fig. 4B, MDA content in G group increased in comparison with that in $\mathrm{C}$ group $(\mathrm{P}<0.001)$, which was markedly decreased by Sestrin 2 inhibition. The antioxidative enzymes SOD and mitochondrial SOD were reduced in $\mathrm{G}$ group $(\mathrm{P}<0.001)$, suggesting that antioxidant capacity was compromised under HG conditions. However, subsequent to Sestrin2 silencing, antioxidant levels increased significantly $(\mathrm{P}<0.001)$. Collectively, the results indicated that downregulation of Sestrin2 protects $\mathrm{H} 9 \mathrm{c} 2$ cells against $\mathrm{HG}$-induced injuries through alleviation of oxidative stress.

Downregulation of Sestrin2 alleviates HG-induced mitochondrial injury in $\mathrm{H} 9 \mathrm{c} 2$ cells. To investigate the effect of Sestrin2 on mitochondrial function, intracellular calcium (Fig. 5A) and mitochondrial membrane potential in $\mathrm{H} 9 \mathrm{c} 2$ cells was measured (Fig. 5B) by flow cytometry. Results demonstrated that the intracellular calcium concentration of $\mathrm{G}$ group was increased compared with $\mathrm{C}$ group $(\mathrm{P}<0.05$; Fig. 5), while the inhibition of Sestrin2 decreased the intracellular calcium level compared with $\mathrm{G}$ and $\mathrm{NC}$ groups $(\mathrm{P}<0.05)$. The mitochondrial membrane potential was decreased under HG conditions, while the inhibition of Sestrin 2 significantly increased mitochondrial membrane potential compared with $\mathrm{NC}$ group $(\mathrm{P}<0.05)$. The MPTP opening was increased in HG status while the inhibition of Sestrin 2 decreased MPTP opening. Furthermore, ATP, caspase-3, and cytochrome $c$ generations were increased in G group, while Sestrin2 downregulation decreased at all their levels. Taken together, these results demonstrated that the inhibition of Sestrin2 ameliorated mitochondrial function under HG status. The maintenance of mitochondrial function is mainly involved in the protective mechanism.

\section{Discussion}

It has been well documented that mitochondrial dysfunction plays a critical role in the development of DCM. Several aspects of common mitochondrial functions have been described as being altered in DCM, including impaired energy metabolism, compromised mitochondrial dynamics, deficiencies in $\mathrm{Ca}^{2+}$ handling, increases in reactive oxygen species production, and a higher propensity for MPTP opening (8). Enhanced oxidative stress, decreased cell viability and impaired mitochondrial function in an in vitro DCM model have been previously observed (7). Increased apoptosis and necrosis of cardiomyocytes were observed in clinical and animal studies in DCM, which was closely related to mitochondrial dysfunction (9). 

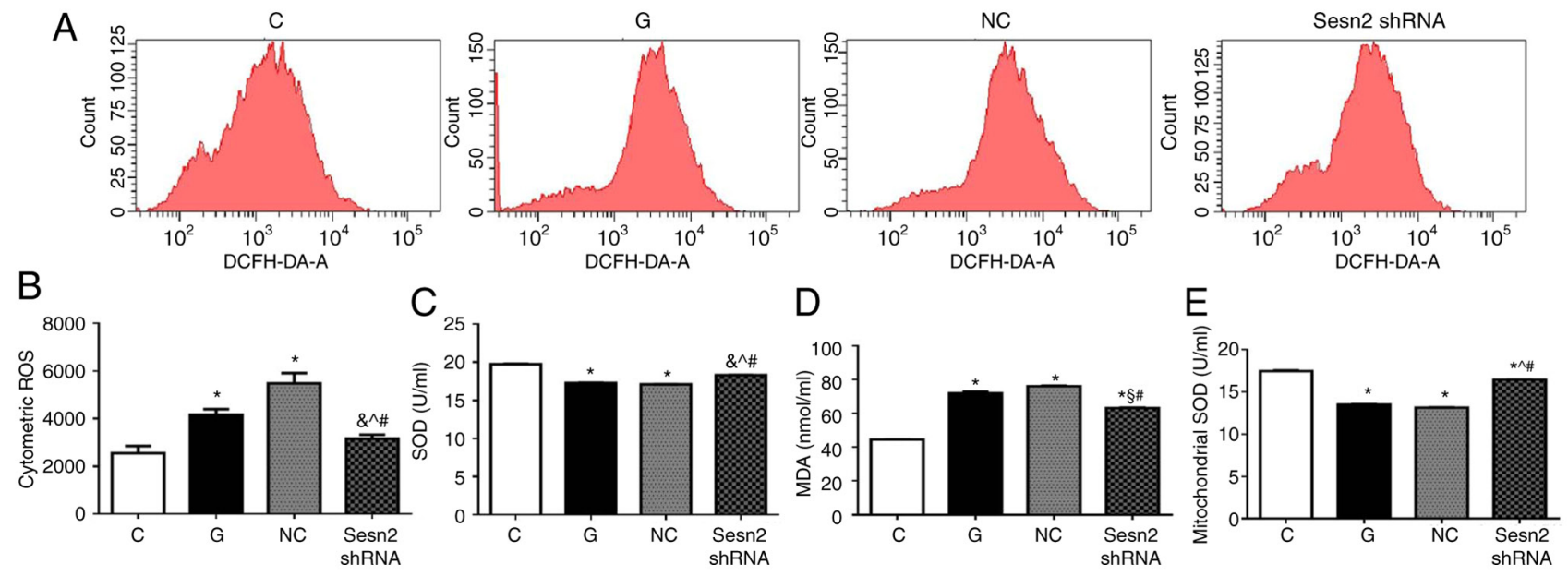

Figure 4. Sestrin2 inhibition significantly decreases oxidative stress in H9c2 cells. (A) Cytometric ROS levels were detected via flow cytometry. (B) Quantitative analysis of ROS levels. (C) SOD, (D) MDA, and (E) mitochondrial SOD kits were used to determine oxidative stress. Data are presented as mean \pm SD ( $=3$ ). ${ }^{*} \mathrm{P}<0.001$ vs. Control group. ${ }^{\star} \mathrm{P}<0.01$ vs. Control group. ${ }^{\wedge} \mathrm{P}<0.001$ vs. HG group. ${ }^{\circledR} \mathrm{P}<0.01$ vs. HG group. ${ }^{\#} \mathrm{P}<0.001$ vs. Negative control group. Sesn2, Sestrin2; C, control group; G, HG group; NC, negative control; shRNA, small hairpin RNA; HG, high glucose.

The present study demonstrated increased cellular apoptosis, enhanced oxidative stress, and impaired mitochondrial integrity in DCM, which was in accordance with results of previous studies (8).

Sestrin2 is an important oxidative stress response protein. It has been indicated that activation of Sestrin2 plays important roles in reducing ROS accumulation, maintaining energy balance, enhancing autophagy, reducing protein synthesis, modulating cell growth, and retaining the progression of metabolic diseases (10). Sestrin2 was reported to be involved in various diseases. Previously, there were some researches which investigated the role of Sestrin 2 in cardiovascular diseases, but mainly in ischemic and age-related heart diseases. Sestrin2 alleviated oxidative stress and ER stress, and activated autophagy in these experimental models (11). In aged hearts, Sestrin2 expression was reduced (12). Additionally, Sestrin 2 knockout enhanced pressure overload-induced cardiac hypertrophy and Sestrin 2 rescue partially alleviated cardiac hypertrophy in aged mice. In research concerning diabetes, Sestrin2 was considered to be involved in maintaining insulin sensitivity. Sestrin 2 deficiency increased obesity-induced insulin resistance and accelerated the progression of diabetes (13). The expression of Sestrin 2 was found to be decreased in podocytes and monocytes in HG conditions. The activation of Sestrin2 ameliorated mitochondrial dysfunction and was involved in the regulation of HG-mediated atherosclerosis $(14,15)$.

To the best of our knowledge, this is the first research to investigate the role of Sestrin2 in DCM. An increased expression of Sestrin2 was identified in DCM models. Considering the previous researches, this finding led to speculation of probable protection effect of Sestrin 2 in DCM. However, beyond our expectation, inhibition of Sestrin2 ameliorated cardiomyocyte injury by alleviating mitochondrial dysfunction, which is in contrast to previous studies in other inflammatory conditions $(11,12,16,17)$.

The levels of Sestrin2 were upregulated or downregulated in different stress conditions. However, in most in vitro and animal studies, the activation of Sestrin 2 was similarly reported to play protective roles while the downregulation or knockdown of Sestrin2 was indicated to aggravate these stress conditions. A study showed that the knockdown of Sestrin2 increased lipopolysaccharide-induced oxidative stress and apoptosis in $\mathrm{H} 9 \mathrm{c} 2$ cells and heart tissues of mice (16). In rats, Sestrin 2 knockdown was reported to aggravate the cardiomyocyte hypertrophy induced by phenylephrine, and Sestrin 2 overexpression protected cardiomyocytes from hypertrophy (17).

Previous findings have shown that the upregulation of Sestrin2 was not suggested to be protective in some conditions. Early researches in Sestrin 2 have proven that overexpression of Sestrin2 full-length cDNA was toxic for many types of cultured cells (18). Clinical studies in Sestrin2 are on the increase in recent years and contradictory results were shown. For example, Sestrin 2 concentrations were increased in patients with chronic heart failure $(\mathrm{CHF})$, and correlated positively with the severity of CHF (19). Upregulated Sestrin2 significantly increased the occurrence of major adverse cardiac events and indicated poor outcome in patients with CHF (19). Similarly, plasma Sestrin2 level in patients with coronary artery disease (CAD) was found to be high and associated with the severity of CAD (20). In addition, serum Sestrin 2 concentration showed an increasing trend in patients with metabolic syndrome. Plasma sestrin 2 concentrations were high in patients with carotid plaque and were associated with plaque severity (21). In particular, concerning the expression of Sestrin2 in diabetes, studies presented inconsistent findings. A study in patients with newly diagnosed T2DM demonstrated a trend for increased Sestrin2 level, which was significantly related to insulin resistance and percentage body fat (22). However, other studies in patients with T2DM and in patients with diabetic nephropathy (DN) reported significantly decreased Sestrin2 levels $(23,24)$. Thus, Sestrin2 seems to be involved in complex regulation mechanisms and reacts differently in different stress conditions. Based on findings of the present study and the aforementioned studies, Sestrin 2 levels may increase in a compensatory manner in response to oxidative stress and exert detrimental effects in DCM. The roles of Sestrin 2 are more complicated than expected. 
A
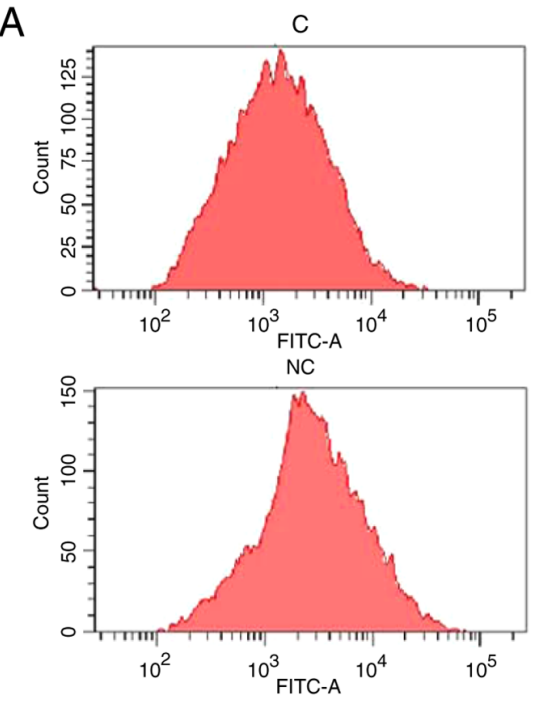

B

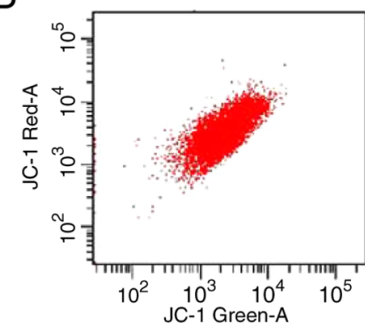

NC

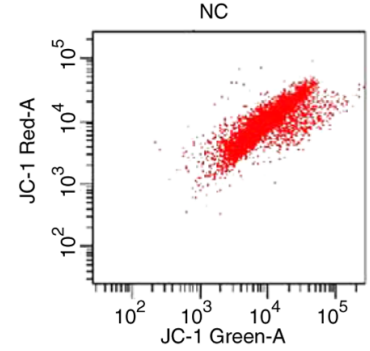

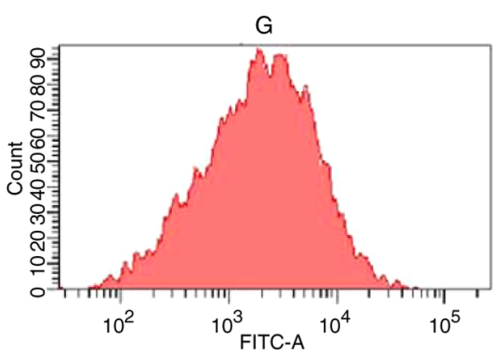

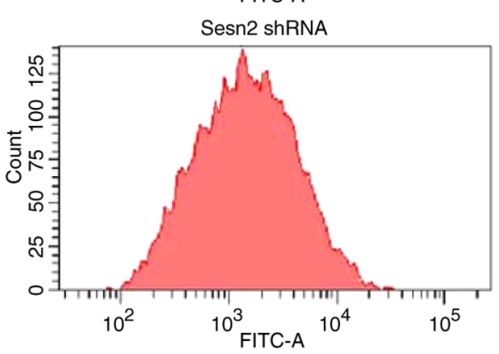

G
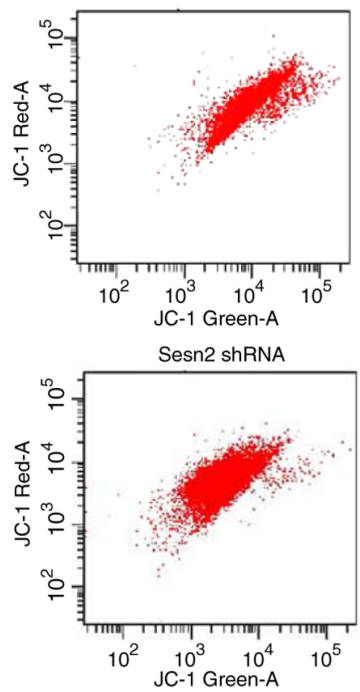

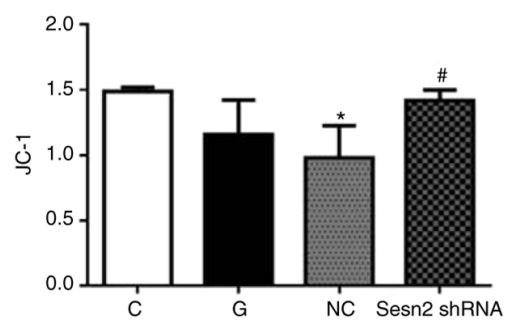

NC
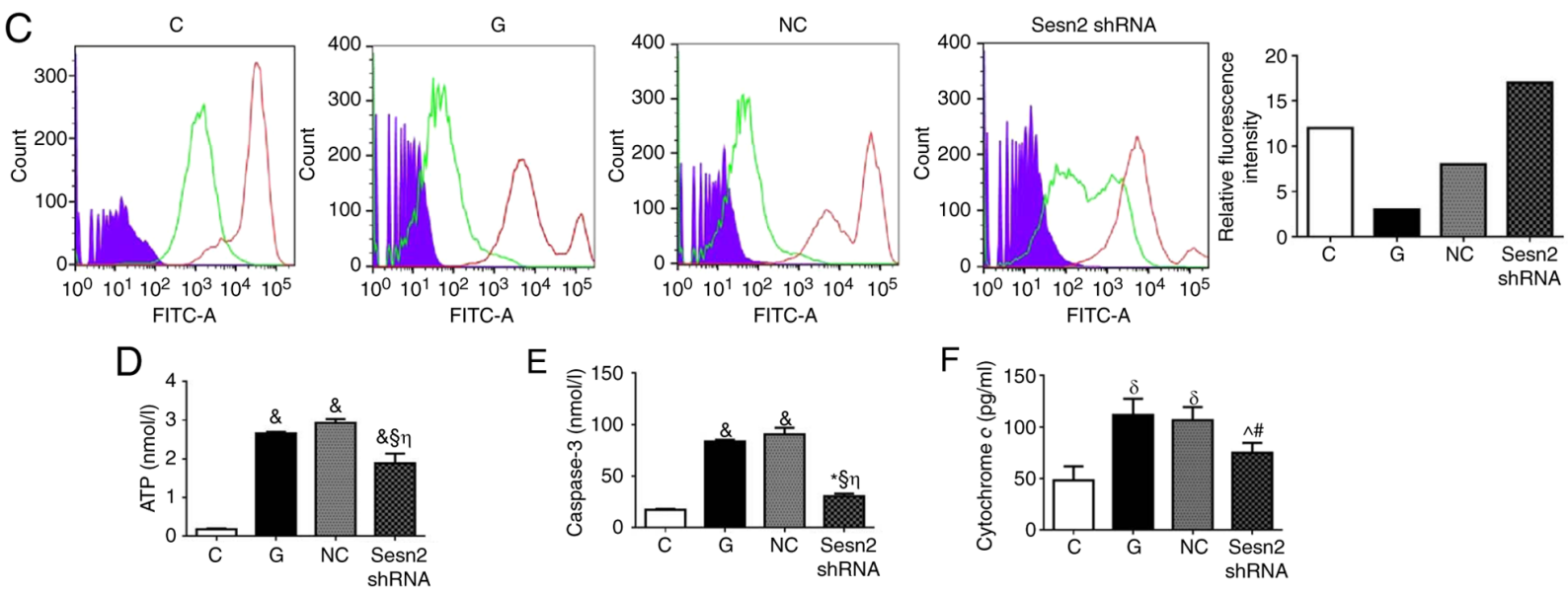

Figure 5. Sestrin2 inhibition restores mitochondrial function in H9c2 cells. (A) Intracellular calcium was assessed by flow cytometry. (B) The mitochondrial membrane potential was measured by flow cytometry. (C) MPTP opening was detected by flow cytometry. The fluorescence intensity was negatively associated with MPTP opening. (D) The level of ATP was measured by ATP assay kit. The activities of (E) caspase-3 and (F) cytochrome $c$ were measured by ELISA. Data are presented as mean $\pm \mathrm{SD}(\mathrm{n}=3) .{ }^{*} \mathrm{P}<0.05$ vs. Control group. ${ }^{\circ} \mathrm{P}<0.01$ vs. Control group. ${ }^{\circledR} \mathrm{P}<0.001$ vs. Control group. ${ }^{\wedge} \mathrm{P}<0.05$ vs. HG group. ${ }^{\S} \mathrm{P}<0.01$ vs. HG group. ${ }^{\text {"P}} \mathrm{P}<0.05$ vs. Negative control group. ${ }^{\eta} \mathrm{P}<0.01$ vs. Negative control group. Sesn2, Sestrin2; C, control group; G, HG group; NC, negative control; shRNA, small hairpin RNA; HG, high glucose.

The present study has several limitations. First, the findings were mainly derived from in vitro research. Second, the molecular mechanisms that connect Sestrin2 and mitochondrial function were not extensively explored. Further investigations are required to clarify the roles and underlying mechanisms of Sestrin2 in DCM. 
In conclusion, the present study demonstrated that inhibition of enhanced Sestrin2 expression ameliorates cardiac injury in DCM, which may be largely attributed to restoration of mitochondrial function.

\section{Acknowledgements}

Not applicable.

\section{Funding}

The present study was supported by grants from the National Natural Science Foundation of China (grant no. 81800726) and the Natural Science Foundation of Guangdong, China (grant no. 2017A030310257).

\section{Availability of data and materials}

The datasets used and/or analyzed during the current study are available from the corresponding author on reasonable request.

\section{Authors' contributions}

$\mathrm{XZ}$ and WL contributed to the conception and design of the study. $\mathrm{XZ}, \mathrm{XD}$ and $\mathrm{HY}$ conducted the experiments. XZ and WL confirm the authenticity of all the raw data. XZ, XD and ZC analyzed the data and wrote the manuscript. XZ and WL reviewed and edited the manuscript. All authors read and approved the final manuscript.

\section{Ethics approval and consent to participate}

All animal procedures were approved by the Ethics Committee of the Second Affiliated Hospital of Guangzhou Medical University (Guangzhou, China) (approval no.: 20200407).

\section{Patient consent for publication}

Not applicable.

\section{Competing interests}

The authors declare that they have no competing interests.

\section{References}

1. Cosentino F, Grant PJ, Aboyans V, Bailey CJ, Ceriello A, Delgado V, Federici M, Filippatos G, Grobbee DE, Hansen TB, et al: 2019 ESC Guidelines on diabetes, pre-diabetes, and cardiovascular diseases developed in collaboration with the EASD. Eur Heart J 41: 255-323, 2020.

2. Jia G, Whaley-Connell A and Sowers JR: Diabetic cardiomyopathy: A hyperglycaemia and insulin resistance-induced heart disease. Diabetologia 61: 21-28, 2018.

3. Lee JH, Budanov AV, Park EJ, Birse R, Kim TE, Perkins GA, Ocorr K, Rllisman MH, Bodmer R, Bier E and Karin M: Sestrin as a feedback inhibitor of TOR that prevents age-related pathologies. Science 327: 1223-1228, 2010.

4. Gao A, Li F, Zhou Q and Chen L: Sestrin2 as a potential therapeutic target for cardiovascular diseases. Pharmacol Res 159: 104990, 2020.

5. Ding B, Parmigiani A, Divakaruni AS, Archer K, Murphy AN and Budanov AV: Sestrin2 is induced by glucose starvation via the unfolded protein response and protects cells from non-canonical necroptotic cell death. Sci Rep 6: 2253, 2016.
6. Livak KJ and Schmittgen TD: Analysis of reactive gene expression data using real-time quantitative PCR and the 2(-Delta Delta C(T)) method. Methods 25: 402-408, 2001.

7. Zhang X, Zhong Z and Li W: Downregulation of TRAP1 aggravates injury of $\mathrm{H} 9 \mathrm{c} 2$ cardiomyocytes in a hyperglycemic state. Exp Ther Med 18: 2681-2686, 2019.

8. Federico M, Fuente S, Palomeque $J$ and Sheu S: The role of mitochondria in metabolic disease: A special emphasis on heart dysfunction. J Physiol 599: 3477-3493, 2021.

9. Kubli D and Gustafsson $\AA$ : Unbreak my heart: Targeting mitochondrial autophagy in diabetic cardiomyopathy. Antioxid Redox Signal 22: 1527-1544, 2015.

10. Budanov AV, Lee JH and Karin M: Stressin Sestrins take an aging fight. EMBO Mol Med 2: 388-400, 2010.

11. Sun W, Wang Y, Zheng Y and Quan N: The emerging role of Sestrin2 in cell metabolism, and cardiovascular and age-related diseases. Aging Dis 11: 154-163, 2020.

12. Quan N, Li X, Zhang J, Han Y, Sun W, Ren D, Tong Q and Li J: Substrate metabolism regulated by Sestrin2-mTORC1 alleviates pressure overload-induced cardiac hypertrophy in aged heart. Redox Biol 36: 101637, 2020.

13. Lee JH, Budanov AV, Talukdar S, Park EJ, Park HL, Park HW, Bandyopadhyay G, Li N, Aghajan M, Jang I, et al: Maintenance of metabolic homeostasis by Sestrin2 and Sestrin3. Cell Metab 16: 311-321, 2012.

14. Lin Q, Ma Y, Chen Z, Hu J, Chen C, Fan Y, Liang W and Ding G: Sestrin-2 regulates podocyte mitochondrial dysfunction and apoptosis under high-glucose conditions via AMPK. Int J Mol Med 45: 1361-1372, 2020.

15. Sundararajan S, Jayachandran I, Balasubramanyam M, Mohan V, Venkatesan B and Manickam N: Sestrin2 regulates monocyte activation through AMPK-mTOR nexus under high-glucose and dyslipidemic conditions. J Cell Biochem: Nov 18, 2018 (Epub ahead of print). doi: 10.1002/jcb.28102.

16. Hwang H, Kim JW, Chung HS, Seo JA, Kim SG, Kim NH, Choi KM, Baik SH and Yoo HJ: Knockdown of Sestrin2 increases lipopolysaccharide-induced oxidative stress, apoptosis, and fibrotic reactions in $\mathrm{H} 9 \mathrm{c} 2$ cells and heart tissues of mice via an AMPK-dependent mechanism. Mediators Inflamm 2018: 6209140, 2018.

17. Dong B, Xue R, Sun Y, Dong Y and Liu C: Sestrin 2 attenuates neonatal rat cardiomyocyte hypertrophy induced by phenylephrine via inhibiting ERK1/2. Mol Cell Biochem 433: 113-123, 2017.

18. Budanov AV, Shoshani T, Faerman A, Zelin E, Kamer I, Kalinski H, Gorodin S, Fishman A, Chajut A, Einat P, et al: Identification of a novel stress-responsive gene Hi95 involved in regulation of cell viability. Oncogene 21: 6017-6031, 2002.

19. Wang H, Li N, Shao X, Li J, Guo L, Yu X, Sun Y, Hao J, Xiang J, $\mathrm{Li} X$ and Han X: Increased plasma sestrin 2 concentrations in patients with chronic heart failure and predicted the occurrence of major adverse cardiac events: A 36-month follow-up cohort study. Clin Chim Acta 495: 338-344, 2019.

20. Kishimoto Y, Aoyama M, Saita E, Ikegami Y, Ohmori R, Kondo K and Momiyama Y: Association between plasma Sestrin2 Levels and the presence and severity of coronary artery disease. Dis Markers 2020: 7439574, 2020.

21. Kishimoto Y, Saita E, Ohmori R, Kondo K and Momiyama Y: Plasma sestrin 2 concentrations and carotid atherosclerosis. Clin Chim Acta 504: 56-59, 2020.

22. Chung HS, Hwang H, Hwang SY, Kim NH, Seo JA, Kim SG, Kim NH, Sei Baik H, Choi KM and Yoo HJ: Association of serum Sestrin2 level with metabolic risk factors in newly diagnosed drug-naïve type 2 diabetes. Diabetes Res Clin Pract 144: 34-41, 2018.

23. Sundararajan S, Jayachandran I, Subramanian SC, Anjana RM, Balasubramanyam M, Mohan V, Venkatesan B and Manickam N: Decreased Sestrin levels in patients with type 2 diabetes and dyslipidemia and their association with the severity of atherogenic index. J Endocrinol Invest 44: 1395-1405, 2021.

24. Mohany KM and Rugaie OA: Association of serum sestrin 2 and betatrophin with serum neutrophil gelatinase associated lipocalin levels in type 2 diabetic patients with diabetic nephropathy. J Diabetes Metab Disord 19: 249-256, 2020.

This work is licensed under a Creative Commons Attribution-NonCommercial-NoDerivatives 4.0 International (CC BY-NC-ND 4.0) License. 